\title{
LA ESCRITURA DE LA TEORÍA COMO AUTOFICCIÓN:
}

\author{
ALAIN ROBBE-GRILLET
}

\section{WRITING THEORY AS AUTOFICTION: \\ ALAIN ROBBE-GRILLET}

\author{
José María Fernández Cardo \\ Universidad de Oviedo
}

\section{ABSTRACT}

In this article we analyze the presence of theoretical discourse and critical theory within Alain Robee-Grillet's autofiction, published between 1984 and 1994 under the title of Les Romanesques. The possibility of representation of the 'I' lays within the duplicity network of intertextual characteristics, which extends from Marcel Proust to Mario Bellatín. It offers an alternative rearrangement of the text which may be included in the autofiction genre, renamed as nouvelle autobiographie. The main purpose of this study is to analyze a way of writing, metaliterary and auto/analytic, which, paradoxically, offers a fictional reading.

Keywords: Alain Robbe-Grillet, Marcel Proust, Mario Bellatin, writing, theory, autofiction, new autobiography, nouveau roman. 


\section{RESUMEN}

Se aborda la cuestión de la presencia del discurso de la teoría y de la crítica literarias dentro de los textos de autoficción de Alain Robbe-Grillet, publicados entre 1984 y 1994, agrupados bajo el título general de Les romanesques. El problema de la posibilidad de la representación del yo se sitúa dentro de una red de duplicidades de carácter intertextual, que se extiende hasta Marcel Proust y Mario Bellatin, y que conduce a una propuesta de ordenamiento graduado de los textos susceptibles de formar parte, con alguna que otra distancia, del género de la autoficción, recalificado de nouvelle autobiographie. El objeto fundamental del estudio lo constituye un modo de escritura de orientación metaliteraria y autoanalítica que, paradójicamente, se ofrece a la lectura como texto ficcional.

Palabras clave: Alain Robbe-Grillet, Marcel Proust, Mario Bellatin, escritura, teoría, autoficción, nueva autobiografía, nouveau roman.

Fecha de recepción: 10 de octubre de 2021.

Fecha de aceptación: 11 de noviembre de 2021.

Cómo citar: Fernández Cardo, José María (2021): «La escritura de la teoría como autoficción: Alain Robbe-Grillet», en Actio Nova: Revista de Teoría de la Literatura y Literatura Comparada, 5: 330-358.

DOI: https://doi.org/10.15366/actionova2021.5.014 
La búsqueda del silencio como expresión de la nada, del vacío, es una aporía: una vez representada la nada por el silencio-figura ya nada queda por hacer: la solución más consecuente es entregarse a la repetición.

Teresa Pereira Romero ${ }^{1}$

\section{0.- INCIPIT}

Los tres términos y el nombre propio presentes en el título solicitan un breve apunte desde el mismo introito del presente artículo. Conviene, en efecto, subrayar que los tres términos en cuestión son palabras mayores dentro de cualquier práctica discursiva relacionada con el ámbito de la Teoría de la Literatura. El lector riguroso pensará con razón que para tratar de la teoría, de la autoficción y de la escritura habría que posicionarse y decir cuál es el sentido en que se emplean esas voces, pero no es mi propósito acotar el significado de las mismas ab initio, sino dejarlas al arbitrio de la significancia de sus ocurrencias «chemin faisant»; no fuera a suceder que hubiera que resituarlas, en función de la contextualización o del uso aplicado que de ellas se haga con relación a los textos que serán objeto de lectura a lo largo de este trabajo.

Bien se diría, por el contrario, que el nombre propio Alain Robbe-Grillet es el de más claro referente, en la medida en que designa a un autor determinado de la serie de la literatura francesa de la segunda mitad del siglo XX y de los primeros años del XXI. Nacido en 1922, su primera novela, Les Gommes, se publica en 1953, y fallece en 2008. De inmediato el lector, al suministrarle estos datos relacionados con la cronología, habrá colegido que hay en este artículo una voluntad conmemorativa, escrito en la antesala del año del centenario del nacimiento del autor, pero no sólo eso, ya que 2022 es también el centenario del fallecimiento de Marcel Proust, inseparable de la genealogía de la narrativa francesa conducente a la nueva novela -nouveau roman-, de la que Robbe-Grillet es representante acreditado. ¡No deja de resultar sorprendente que 1922 sea el año de nacimiento de Robbe-Grillet y el de fallecimiento de Marcel Proust, y mucho más dentro de un trabajo como éste, provisto de un título particularmente adecuado para ilustrar una gran parte del movimiento creativo que

\footnotetext{
1 Silencio y Poesía. La obra de Yves Bonnefoy (1993: 62).
} 
contiene $\dot{A}$ la recherche du temps perdu, obra por excelencia en la que se concitan a través de la escritura la teoría literaria y la ficción autobiográfica!.

\section{1.- LA SERIE DE LOS ROBBE-GRILLET O LA REFERENCIA DILUIDA}

Corresponde a Roland Barthes el mérito de haber puesto en tela de juicio la unidad de la signatura Robbe-Grillet, su capacidad para designar de modo unívoco el grupo de textos que la firma reúne. Naturalmente no es éste un caso singular en la historia de la recepción de la obra literaria de un autor, que una tradición bien establecida al respecto ha tratado de presentar casi siempre como conjunto homogéneo, por mucho que se admita una evolución endógena. Efectivamente fue Roland Barthes el que, en 1963, diez años después de la publicación de la primera novela del autor, empezó a hablar de Robbe-Grillet $\mathrm{n}^{\circ} 1$ y nº2, dentro del famoso prólogo, al menos para la crítica especializada, del libro de Bruce Morrissette Les romans de Robbe-Grillet (1963). Quería insistir Roland Barthes en las diferencias de las dos lecturas sucesivas de sus novelas, una de orientación «objetivista» y otra de orientación «subjetivista». Por aquella misma época, a principios de la década de los sesenta, se había establecido ya una oposición de otro tenor en la recepción de Robbe-Grillet, Teórico/Escritor ( $\mathrm{n}^{\circ} 3$ y $\left.\mathrm{n}^{\circ} 4\right)$, porque no conviene olvidar que el nouveau roman desde sus orígenes navegaba entre dos aguas, un discurso crítico-teórico y una praxis novelística, que el mismo Roland Barthes se encargó de hacer confluir al afirmar de modo contundente que no hay grandes diferencias entre los considerados escritores y los críticos literarios, porque ambos comparten una misma pasión que los reúne, el sentido del lenguaje: si para el escritor el lenguaje representa un problema, consciente de su profundidad, más allá de su valor instrumental, no es menos cierto que el discurso del crítico -de la teoría- comparte la misma preocupación (Critique et Vérité, 1966).

$\mathrm{Al}$ periodo del nouveau roman, al menos en la historiografía literaria francesa sobre la materia, le sucedió el período del nouveau nouveau roman, denominación con la que se trataba de designar una segunda época dentro de la producción novelística del grupo y de la de Robbe-Grillet en particular. Si el primero se caracterizaba por la amenaza a la unidad del relato, el segundo, para enunciarlo de manera sintética, por la unidad imposible o pérdida de la misma, y en el caso del autor que me ocupa esta tendencia se habría manifestado sobre todo a partir de La maison de rendez-vous (1965), para continuar con Projet pour une révolution à 
New York (1970) y varias novelas más, hasta adentrarse en el siglo XXI con las dos últimas, La reprise (2001) y Un roman sentimental (2007) - lo que nos colocaría ante el Robbe-Grillet nº5 de la serie.

Pero todavía hay más. La década de los ochenta acoge una denominación distinta para designar las obras del mismo grupo de escritores, entre los que se cuentan también Marguerite Duras y Claude Simon. Surge el calificativo de nouvelle autobiographie: los novelistas del nowveau roman irrumpen en el género de la autobiografía de una manera muy distinta a la que Philippe Lejeune había descrito y codificado en el famoso Pacte autobiographique (1975), que, podría decirse, se ocupaba en ese libro de la antigua, al menos desde la perspectiva del Robbe-Grillet que inicia la publicación, desde mediados de esa década, de Le miroir qui revient (1984), Angélique ou l'enchantement (1988) y Les derniers jours de Corinthe (1994), tres volúmenes agrupados bajo el subtítulo genérico de Romanesques (I,II y III), su contribución a la nueva manera de discurso autobiográfico - o lo que a efectos de este cómputo seriado que aquí se viene practicando, y que bien puede tomarse por osadía crítica sin precedentes, nos vendría a colocar ante el Robbe-Grillet $n^{\circ} 6$.

Y queda todavía el n ${ }^{\circ} 7$. No es fácil su caracterización, ni siquiera la adjudicación de un nombre conveniente, a pesar de su pertinencia a los efectos de la autoficción o quizás de la pura y simple biografía, mucho menos pretenciosa como materia de literatura, que se expresa a través de un proceso de textualización no habitual, o por lo menos inesperado dentro del proceso de recepción de un autor inseparable de cierta mitología de lo que se ha dado en nombrar «la modernidad», utilizada aquí con la ambigüedad referencial que le es propia. Robbe-Grillet $\mathrm{n}^{\circ} 7$ estaría hecho de o representado por textos varios, diversos, pero significantes a efectos de la causa biográfica. Entre ellos, cabría señalar. en primer lugar, el libro publicado en 2005 Alain Robbe-Grillet, préface à une vie d'écrivain. La formulación del título no puede pasarnos desapercibida, merece subrayar el término que matiza y restringe el enunciado: no se trata de la vida del escritor, sino del prefacio, el material previo (biográfico y teórico) que habría contribuido a modelar la vida del escritor. La enunciación sucede en primera persona, pero no se trata de un texto escrito, sino de la reproducción escrita de un texto oral, en el que se recogen las sucesivas intervenciones de Robbe-Grillet en una emisión seriada de radio, emitida por France Culture en el verano de 2003. El editor (publicado conjuntamente por Seuil y France Culture, Fiction \& Cie) presenta el libro como versión complementaria o vade-mecum del CD MP3 adherido a la solapa en la página interior de la contraportada, que contiene las doce horas de emisión. Se ha pretendido, pues, respetar de 
forma escrupulosa el carácter oral del material original. Ni que decir tiene que se trata de una compilación de primera importancia sobre aspectos biográficos y declaraciones teóricas de Robbe-Grillet, que, en su mayor parte, tampoco deberían sorprender a la crítica especializada, conocedora de las intervenciones orales de un autor que no ha tenido empacho en prodigarse a través de coloquios, entrevistas y clases en las universidades del mundo (europeas, americanas e incluso asiáticas).

Material complementario de carácter biográfico, susceptible de ser incluido en ese mismo apartado $\mathrm{n}^{\circ} 7$, lo constituyen dos publicaciones posteriores a la muerte del autor (2008), ambas de 2012, y en las que Emmanuelle Lambert ha tenido un papel primordial, Correspondance 1951-1990, en la que figuran como autores Alain y Catherine Robbe-Grillet (esposa del escritor y escritora ella misma), volumen de cerca de setecientas páginas (Fayard) en el que fluye directamente la palabra escrita, en la forma habitual de la correspondencia; $y$, la segunda de ellas, Alain, libro singular, escrito por la mencionada esposa del escritor, Catherine, publicado igualmente por Fayard, y sobre todo por su estructura: 51 artículos ordenados por orden alfabético, de la $\mathrm{A}$ a la Z, en que se proporciona información biográfica sin tapujos, incluso relacionada con aspectos íntimos de la vida cotidiana -la sexualidad no se excluye-. Los títulos de los artículos de este singular diccionario biográfico remiten en su mayor parte a objetos comunes de la vida cotidiana, como Baignoire, Cuisine, Lit, Mouchoirs, Verre à eau, Voiture, o Yaourt ...

$\mathrm{Y}$, finalmente, en el uso y abuso del libertinaje en la aplicación disgregadora de los dígitos numéricos sucesivos para señalar la pluralidad de la obra, quedaría el Robbe-Grillet $\mathrm{n}^{\circ} 8$, a sabiendas de que este número es fundacional, o casi privilegiado, dentro de la misma, por su capacidad emblemática de expresar los fantasmas obsesivos del autor a partir de Le Voyeur (1955), novela de la escritura de la mirada, y manifestación inaugural de su fascinación por la imagen, que dará lugar a la ristra de películas entre las que figuran títulos como L’homme qui ment (1968), significante también dentro del tema de este trabajo, L'année dernière à Marienbad (1961, con Resnais), L'Eden et après (1971), Glissements progressifs du plaisir (1974) o C'est Gradiva qui vous appelle (2006), a sabiendas de que ninguna de ellas constituye adaptación de ninguna de sus novelas. 


\section{2.- A vUELTAS CON LA TEORÍA, DE LA NOVELA Y DE LA LitERATURA.}

Se impone ahora, como punto de partida de este nuevo apartado, por mor de coherencia textual y metodológica, recuperar la serie aludida arriba de Robbe-Grillet $\mathrm{n}^{\mathrm{0}} 3$ y Robbe-Grillet $\mathrm{n}^{\circ} 4$, referida a la pareja Teórico/Escritor, que quizás convendría formular no ya en términos de oposición, como parece indicar la barra separadora, sino en los de una coexistencia conjunta mediante guion, como Teórico-Escritor (Teoría y Escritura, Metalenguaje y Lenguaje), sin dejar de señalar que aquí, como en la multiplicación, el orden de los factores no puede ni pretende alterar el producto: el orden de la formulación no quiere decir que la teoría sea primero y después venga la escritura, a modo de subproducto segundo. En definitiva, aquí se da por buena la hipótesis esbozada por Henri Meschonnic (1970: 164) en el sentido de que, desde la modernidad, habría que considerar estéril y no productiva la separación impuesta por la ideología al uso entre lenguaje y metalenguaje, porque, según él, la experiencia prueba más bien lo contrario: los más grandes en la práctica son también los más grandes en la teoría. El término acuñado por Gilles Deleuze (1969) de la disyunción inclusiva podría ser invocado también aquí a los efectos de nombrar de forma conveniente la reunión de la teoría y de la práctica de la escritura; tutela conceptual, si se quiere, para un tipo de discurso como éste, marcado por el vaivén entre la una y la otra, que trata de ser así mismo coherente con la formulación barhesiana, mencionada en uno de los párrafos precedentes, en la que se reivindicaba el papel de la crítica como acto de escritura, la no separación de la actividad del crítico y la del escritor (ambos sienten el lenguaje como problema).

Robbe-Grillet dice de manera sencilla que, cuando se interesa por una cosa, se interesa por la teoría de la cosa; si se interesa por las plantas, de inmediato se interesa por la botánica, y lo mismo le ocurre con la literatura: «Si je m’intéresse à la littérature, je vais être amené à m’intéresser à l'histoire de la littérature, et donc aux différentes théories qui ont jalonné l'histoire de la littérature: théories que je ne prends pas du tout comme des vérités, mais comme des hypothèses $»^{2}$. Insiste de modo particular en que las teorías no son verdades sino hipótesis; no duda en acudir a su propia experiencia de científico, a su trabajo como ingeniero agrónomo que precedió al de escritor -sería la suya una vocación tardía, cerca de los treinta años-: «Pour moi, comme pour tout scientifique en général, la théorie est une hypothèse. Ce sont les littéraires qui croient que la théorie est vraie. La théorie n’est pas une 
vérité mais un moyen d'investigation sur le réel $»^{3}$. La teoría concebida de esta manera, como hipótesis no necesariamente verdadera, como simple medio de investigar lo real, no estaría tan alejada de algunos de los postulados que caracterizan la ficción, y, en consecuencia, sería ella misma susceptible de ser integrada en relatos ficcionales, entre los que cabe incluir también los autoficcionales. De todos modos, y si lo que interesa es el contacto y la imbricación de los dos tipos de discursos de la misma rúbrica (de un mismo autor), el de la ficción y el de la teoría, es obvio que el reconocimiento del discurso de la teoría está asegurado cuando se presenta aislado, publicación como texto independiente, $y$, en menor grado, si se encuentra a modo de fragmento diluido dentro de un conjunto ficcional del que forma parte. Otro tipo de relación diferente, y de carácter bastante más complejo (podría nombrarse intertextual), es el que cabe establecer, a través de la lectura, de la copresencia de la teoría en un texto del mismo autor que se ofrece lisa y llanamente como ficción, simple obra de lenguaje, una novela, por ejemplo, que no utiliza para nada el metalenguaje. La casuística que acaba de ser evocada en abstracto encontraría aplicaciones varias en el conjunto de la obra de Robbe-Grillet, cuya ilustración requeriría análisis particulares que ahora no vienen al caso... Bastaría quizás con traer a colación Bouvard et Pécuchet de Flaubert o Les Faux-monnayeurs de Gide para ejemplificar algunos de los procedimientos aludidos, de la misma o parecida naturaleza.

Además de Préface à une vie d'écrivain, mencionado arriba, dos son los libros fundamentalmente «teóricos» de Robbe-Grillet, Pour un nouveau roman y Le voyageur, de características muy diferentes y separados en fecha de publicación por casi cuarenta años, el primero en 1963 y el segundo en 2001. En efecto son libros de teoría, o más bien de teorías, pero de naturaleza muy diferente. El primero, Pour un nouveau roman, puede ser considerado una especie de manifiesto hecho de la reunión de varios artículos diseminados en revistas que se ocupaban de la crítica literaria de la literatura contemporánea en la década de los años cincuenta y en los primeros años de la siguiente (L'Express, France-Observateur, La NRF, Tel Quel, Critique). Especie de manifiesto porque defiende la causa del nowveau roman frente a las consideradas novelas de factura y estructura «tradicionales», y hermanado por sus objetivos con otros dos libros publicados por otros dos representantes del grupo de los nuevos novelistas, L'ère du soupçon (1956) de Nathalie Sarraute y Essais sur le roman (1964) de Michel Butor.

\footnotetext{
${ }^{3}$ Intervención de Robbe-Grillet en la emisión radiofónica «Jeu de Paume», el 22 de septiembre de 1998. Texto citado por Simonin, 2000: 422.
} 
Le Voyageur (551 páginas) es un libro de teoría bien distinto, bien se diría de madurez, para un escritor que ya ha recorrido la mayor parte del camino, dividido en dos grandes partes. La primera, la más extensa, ocupa algo más de la mitad del libro, se titula TEXTES ET CAUSERIES, y la segunda ENTRETIENS (textos y habladurías, la primera; entrevistas la segunda). Roland Barthes, Honoré de Balzac, Gustave Flaubert y Claude Simon son los autores que aparecen citados con un mayor índice de frecuencia, además de Jean Paul Sartre, Albert Camus, Frank Kafka, Nathalie Sarraute, Marguerite Duras, Marcel Proust o el marqués de Sade, o incluso poetas como Stéphane Mallarmé o Paul Valéry. El concepto de la representación en la novela (el realismo), la relación de la literatura y la política, el cine y la ideología, el ejercicio problemático y la noción de la literatura, el yo del escritor, la autobiografía, la cuestión del doble son algunos de los grandes temas relacionados con la teoría diseminados a lo largo del medio millar de páginas que constituyen el volumen. Hay un solo texto de la década de los cuarenta, muy pocos de la siguiente, la de los cincuenta, algunos de la de los sesenta; la mayor parte de los textos y entrevistas recogidos en el libro pertenecen al período que va de 1970 a 2000. Bien podría, pues, ser considerado Le V oyageur como la corriente teórica que fluye junto a los períodos del nouveau nouveau roman y de la nowvelle autobiographie.

\section{3.- LA PRAXIS CRÍTICA Y EL AFIANZAMIENTO DE LA TEORÍA}

La actividad de Robbe-Grillet escritor es inseparable de una editorial, Les Éditions de Minuit, y de su director, Jérôme Lindon (1925-2001). E incluso cabría señalar que buena parte de la conciencia crítica y teórica sobre la novela de Robbe-Grillet va a afianzarse a lo largo de los treinta años durante los que desempeñó la función de consejero literario de Les Éditions de Minuit. El lanzamiento del nouveau roman está indisociablemente ligado a la figura de Jérôme Lindon y a la editorial de la que era propietario: Samuel Beckett, Nathalie Sarraute, Claude Simon, Marguerite Duras, Michel Butor, Jean Ricardou o Robert Pinget, además del propio Robbe-Grillet, publican, al amparo de ese sello y en momentos clave de sus respectivas carreras literarias, algunos de los títulos que los identifican en mayor medida. Sin duda alguna, los artífices de la irrupción de los nuevos novelistas en las letras francesas en la década de los cincuenta fueron el propietario y el consejero literario de la casa ubicada en el 
número 7 de la rue Bernard Palissy, en cuya puerta está hecha precisamente la foto, recogida en las historias y manuales de la literatura, que representa a los miembros del grupo.

Pero si en este artículo se dedica un breve apartado a la actividad crítica de RobbeGrillet, en su calidad de lector y consejero literario de Les Éditions de Minuit, es para poner de manifiesto que el período comprendido entre 1955 y 19594, fundamental en la génesis de la teoría y de la escritura del nouveau roman, se corresponde con una febril actividad lectora de nuestro autor. Como testimonio fiel de esa actividad han quedado seiscientas cincuenta fichas, escritas por él durante el periodo mencionado, a propósito de los manuscritos que se recibían en la editorial solicitando la publicación. El trabajo de Anne Simonin (2000), que lleva por título «La mise en épreuve du nouveau roman. Six cent cinquante fiches de lecture d'Alain Robbe-Grillet (1955-1959)» ${ }^{5}$,constituye una valiosísima contribución a efectos de ventilar lo que, en tantas ocasiones, constituye la zona obscura de los procesos editoriales, el espacio de lo que Didier Coste (1988) llamaba la «historia literaria negativa», refiriéndose a los inéditos escritos, y como tales integrantes de la historia de la producción literaria, pero que no llegaron nunca a salir a la luz.

En 1959 Robbe-Grillet había publicado cuatro novelas, Les Gommes (1953), Le Voyeur (1955), La Jalousie (1957) y Dans le labyrinthe (octubre de 1959), y escrito otra, inédita por aquellos años, Un régicide. Su elección estética se había puesto ya claramente de manifiesto a través de su praxis como novelista, y la formulación de la teoría, expresada en artículos de prensa y revistas especializadas, había corrido en paralelo cronológico (me refiero a los textos teóricos recogidos más tarde, en 1963, en el volumen arriba citado Pour un nouveau roman; el primero de ellos es de 1953). En definitiva, lo interesante del análisis de Anne Simonin es que permite relacionar las tomas de posición estética del «chef de file» del nouveau roman y su reflejo en las notas escritas sobre las novelas de los otros, su ejercicio de la crítica en función de un determinado canon estético, respaldado y promocionado por él mismo y por Jérôme Lindon. Las razones esgrimidas para la no publicación de los manuscritos vendrían a evidenciar su propio ideario en materia de teoría de la novela en aquella segunda mitad de la década de los cincuenta: rechazo del psicologismo, de la novela regional o de la novela naturalista, en las que la escritura en sí no constituía problema. Alain, el ingeniero agrónomo, esbozaba de esa manera, a través de sus comentarios, una singular botánica que daba lugar a

\footnotetext{
${ }^{4}$ En este período se sitúa una parte importante de la correspondencia de los escritores del grupo nouveau roman, es su momento álgido, «le moment nouveau roman» se denomina en el libro, publicado en fecha reciente, 2021, por Gallimard (NRF), que lleva por título Nouveau Roman. Correspondance 1946-1999. 5 Annales. Histoire, Sciences Sociales. 55e année, 2, 2000: 415-437.
} 
una original clasificación de las especies literarias (Simonin: 418). El tono de las aserciones recogidas en aquellas fichas queda reflejado en el ejemplo reproducido a continuación:

Femme, Paris. Un cas de force majeure

Roman psychologique type dans un décor de haute montagne. Pureté des cimes et ambiguïé des sentiments. Joie des ascensions difficiles.

Accidents de cordée et aléas des histoires d'amour. Pas mal si on veut. À renvoyer sans commentaires [décembre 1957] ${ }^{6}$

Parece, pues, evidente que esas seiscientas cincuenta fichas pueden efectivamente ser consideradas como expresión del « primer estado de la teoría que acompaña la emergencia de una nueva estética novelística $»^{7}$, como era por entonces la del nowveau roman.

\section{4.- Las máscaras (Personae) Del Crítico: Proust y Robbe-Grillet, la} AUTOFICIÓN EN GRADO CERO.

\section{4.- 1.- MARCEL PROUST}

Es en marzo de 1921, el año antes de su fallecimiento, cuando Marcel Proust, que a la sazón luchaba él mismo contra el paso del tiempo biológico y se ocupaba del volumen destinado a clausurar la obra de su vida, Le temps retrouvé, escribe un texto absolutamente singular, desconocido hasta su publicación en 2014, dentro del libro de Michel Schneider L' auteur, l'autre. Proust et son double. Se trata nada más y nada menos que de un texto de crítica literaria escrito por el mismísimo Marcel Proust sobre su propia obra, que lleva por título L' esthétique de Marcel Proust.

Las circunstancias de la escritura y de la no publicación coetánea darían lugar a un larguísimo relato y a toda una serie de análisis críticos, que es lo que precisamente ha nutrido las más de 300 páginas del libro de Michel Schneider, donde se pone de manifiesto una gran capacidad para contextualizar en el universo proustiano un texto de características tan singulares: un autor de la talla de Marcel Proust escribe, para ser publicado con el nombre de otra persona, un texto crítico sobre la propia obra, en un claro ejercicio de autofingimiento, que en el presente apartado ha sido calificado de autoficción en grado cero.

\footnotetext{
${ }^{6}$ Texto de una de las fichas escritas por Robbe-Grillet (Simonin: 422).

7 Traducción mía del original en francés (Simonin: 437).
} 
Para no dejar del todo al lector interesado con una exacerbada sensación de curiosidad, me limitaré a recordar brevemente algunas de las circunstancias. Un joven crítico, de veinte años de edad, llamado Thiébault Sisson, con el fin de escribir un artículo sobre su obra, solicita a Proust que le conteste por carta a una serie de preguntas. Marcel Proust, después de darle algunas vueltas al asunto, tal como se recoge en la correspondencia, le propone al joven crítico enviarle un texto escrito por él mismo, a condición de que no figure el nombre del autor real y para que se publique con la firma del propio Thíébault Sisson. Todo habría sido perfecto si no hubiera sucedido un imprevisto: el fallecimiento inesperado del joven crítico. Un amigo de este, Christian Melchior-Bonnet, se encarga de comunicarle a Proust la desdichada noticia, y a partir de aquí el texto L'esthétique de Marcel Proust pasa al patrimonio de la familia Melchior-Bonnet y de unos herederos que, andando el tiempo, otorgarán a Michel Schneider la autorización para que lo publique.

En origen, L'esthétique de Marcel Proust, era un texto escrito a una sola cara que ocupaba ocho páginas, en papel amarillo de cartas. La última página está cortada en la parte inferior; cabe la posibilidad de que Proust lo hubiera firmado sin darse cuenta ${ }^{8}$. En la edición impresa, tal como se recoge en el libro L'auteur, l'autre. Proust et son double, ocupa cuatro páginas y tres líneas (Schneider: 91-95).

Se inicia el texto con algunas consideraciones relativas a la recepción de Marcel Proust en el extranjero, antes de haber recibido el reconocimiento del premio Goncourt en 1917, en Suiza, Bélgica, Inglaterra y Suecia, además de señalar el interés de un escritor importante por la obra como es el caso de Henry James. Más adelante, se recoge la opinión vertida por un francés, François Mauriac, que había escrito «Nous sommes persuadés que de tous les ouvrages contemporains, ceux de Marcel Proust possèdent les chances les plus grandes de survie», en tanto que renovador del género de la novela. Insiste después en los problemas de los lectores para representarse la construcción de una obra concebida con varias entradas («sextuples porches»), pero pensada como unitaria desde la primera línea hasta la última. El texto crítico insiste sobre todo en el hecho de que los personajes no se desvelan desde el principio, sino más bien al final... La cuestión del estilo de Marcel Proust, tratada con estrategia inteligente de forma diferida a través de citas varias y el enunciado de alguna paradoja, podría suscitar en críticos y lectores alguna reserva. La justificación habría que buscarla en la enfermedad de un escritor que, bien dotado para el estilo, tuvo que

8 Schneider, 2014. 
renunciar a una existencia mundana y retirarse como Monluc ${ }^{9}$ o Montaigne, teniendo que soportar el dolor de los periodos de inactividad. Siguen consideraciones acerca de las relaciones entre el yo del narrador y el yo del autor, a las que se añade una nueva cita de otro francés ilustre, el escritor y crítico Jules Lemaître, que sirve como clausura del texto y juicio de valor: «Ce Proust dans les moments où il est mauvais, c'est tout de même aussi bien que Dickens. Et dans les moments où il est bon c'est tellement mieux! $»^{10} \ldots$ El texto, como bien puede deducirse de la lectura de las líneas precedentes, no se deja resumir con facilidad, es un ejercicio de escritura entrelazada que se ocupa de una serie de asuntos bien conocidos hoy por la crítica proustiana especializada. ¡Imposible de encontrar en él la revelación del único gran secreto inspirador de la obra, que seguramente no existe, por estar diseminado en el conjunto de su factura, en la mezcla de teoría y ficción que ha supuesto el ejercicio de su escritura! Nos quedaremos, pues, con la «inocencia» asertiva, a pesar de la madurez, de un autor que quiso plasmar en un texto generalista escrito con premura, L'esthétique de Marcel Proust, el sentido de una obra inabarcable. No es extraño, pues, que Michel Schneider, en su libro, haya diseminado los componentes de ese texto, obra del autor disfrazado de crítico, y los haya asociado a otros pasajes de la ficción de la obra proustiana, significantes en la misma o parecida medida.

\section{4.- 2.- Proust Y RoBBE-GRILLET ${ }^{11}$}

Dentro de la serie de los novelistas indisociables de la genealogía del nouveau roman, junto a Flaubert, Joyce, Faulkner y Kafka, figura siempre Marcel Proust, pero sabemos que, en el caso particular de Alain Robbe-Grillet - y ciertamente resulta sorprendente- el descubrimiento del autor de $A$ la recherche du temps perdu, es decir su lectura, no sería tan temprana como cabría suponer. El primer contacto sosegado -en $1979^{12}$ - de Robbe-Grillet con la obra proustiana fue Contre Sainte-Beuve, libro de teoría, de imaginación y de autoficción, lo que ciertamente no está carente de significado dentro de su trayectoria literaria personal.

\footnotetext{
${ }^{9}$ Escritor y soldado francés (guerras de Italia y de religión), nacido a principios del siglo XVI y fallecido en 1577, promovido en 1574 a la dignidad de mariscal de Francia y conocido por sus Commentaires, referidos al largo período que va de 1521 a 1576.

10 Schneider, 2014: 95.

11 Robbe-Grillet, en 1998, interpreta el papel de Edmond de Goncourt en la película de Raoul Ruiz Le temps retrouvé.

12 «Question culture, j’ai lu pas mal au mois d'octobre, en particulier les premiers dialogues de Platon [...] Le hasard et la necessité de Monod [...], Les mots de Sartre et le Contre Sainte-Beuve de Proust » (Alain et Catherine Robbe-Grillet, 2012 : 528).
} 
A propósito de ese libro diría Robbe-Grillet sin ambages, nueve años más tarde, en 1988: «Le livre de lui [Proust] qui me passionne le plus, Contre Sainte-Bewve, mélange, comme Le Miroir qui revient, la théorie de la littérature, l'autobiographie et la fiction» ${ }^{13}$.

Catherine, todavía en la década de los cincuenta, en 1954, le había recomendado a Alain Robbe-Grillet, que ya había publicado el año anterior su primera novela, Les gommes, la lectura de Proust: «J'ai beaucoup aimé l' Amour de Swann [...] Je suis effectivement fort étonnée que tu n'aies pas encore lu Proust [...] L'Amour de Swann est un chef-d'œuvre, que je m'aimerais beaucoup que tu lises, pour avoir ton appréciation» (Alain et Catherine RobbeGrillet, 2012: 77).

Gracias a la correspondencia de Alain y Catherine puede datarse con precisión la lectura atenta, continuada e íntegra ${ }^{14}$-lo que no presupone que antes no hubiera tenido un conocimiento parcial-, realizada por Robbe-Grillet de $A$ la recherche $d u$ temps perdu: es en el año 1982, durante una de sus estancias como «profesor de sí mismo» en una universidad de Estados Unidos, en la de Florida. La datación tiene su interés por que será en 1984 cuando Robbe-Grillet va a publicar la primera de sus Romanesques, Le miroir qui revient, obra de autoficción o primera muestra de la nowvelle autobiographie. Y será precisamente con motivo de la publicación de la segunda, en 1988, Angélique ou l'enchantement, cuando las referencias a Proust y a La Recherche aparezcan con mayor insistencia; el modelo de Albertine disparue, sobre el que dice haber reflexionado en fecha reciente, es mencionado al objeto de explicar el funcionamiento del texto autobiográfico que él mismo acaba de publicar, en la respuesta dada a la pregunta realizada por Jacques Henric, recogida en febrero de 1988 en el nº122 de la revista Art Press:

Ça m'amuse que tu me poses cette question parce que je me la suis moi-même posée récemment à propos de Proust. Dans Albertine disparue, Proust se met à réfléchir, dans le texte même, sur la façon dont il est en train d'inventer celui-ci. Il explique sa façon de faire, comment il arrange les choses, corrige pour les besoins de sa démonstration. Il dévoile que ce qu'il a organisé jusqu'alors, c'était dans le but de prouver quelques idées, qu'il avait sur le temps et sur le roman. Le lecteur est à la fois content -il trouve la démonstration intelligente- et frustré. Et curieusement ce sentiment de contentement- frustration qui apparait à la lecture de ces pages-là disparaît à la fin du livre parce que tout été vite repris dans l'océan

\footnotetext{
${ }^{13}$ Respuesta de Robbe-Grillet en la entrevista realizada por Jean-Jacques Brochier publicada en Éditions La Manufacture dentro del libro Alain Robbe-Grillet, colección «Qui suis-je?» en 1985, recogida en Le Voyageur (Robbe-Grillet, 2001: 465).

14 «J'ai absorbé déjà 1400 pages du Proust Pléiade (j’arrive donc du côté de Sodome), mais je finis par faire ce que je m'étais bien promis d'éviter : : lire certaines pages de considérations légèrement en diagonale » (Alain et Catherine Robbe-Grillet, 2012 : 583).
} 
proustien en mouvement. Les pages en question ne sont plus, pour le lecteur, qu'un des éléments de l'océan, c'est-à-dire le projet directeur de quelqu'un qui n'a été lui-même qu'un des épisodiques personnages du roman. La lecture que tu as faite d'Angélique relève de cette sorte de participation active (Robbe-Grillet, $L e$ Voyageur, 2001: 488-489).

Y será también con motivo de la génesis de la tercera, Les derniers jours de Corinthe, cuando de nuevo Robbe-Grillet vuelva a reflexionar una vez más sobre aspectos determinantes de la gran obra proustiana, como es la cuestión de las ediciones post-mortem de la misma. Robbe-Grillet, escritor cuidadoso de las formas en sus detalles más nimios, refiere, dentro de una carta a Catherine en 1987, que acaba de tener la revelación de lo que será la tercera de sus Romanesques, que ha escrito seis páginas de notas, pero que no tiene intención de terminar el libro antes de dos años -en realidad se convertirían en siete, el libro sale en 1994-. Ello le da pie para volver a traer a colación Le temps retrouvé, el último volumen de La Recherche, a modo de contraste con su propia actitud, en exceso vigilante durante el proceso de escritura: «Je passe une grande partie de mon temps avec La Recherche, bien que mon enthousiasme proustien ait, de nouveau, nettement faibli. Toute la seconde moitié du Temps retrouvé est absolument informe : comme tu le sais le texte a été reconstitué à partir de manuscrits divers $[. .$.$] et accompagnés des feuilles volantes que personne ne sait où placer »$ (Alain et Catherine Robbe-Grillet, 2012 : 653). Una vez más vuelve a hacerse presente, en el paratexto robbe-grilletiano, la obra proustiana, de referencia parece que obligada siempre que se trata de la escritura de la autoficción que son Les romanesques. No debería ser obviada esta copresencia ni ser considerada insignificante... Pero, puesto que de lo que en el presente apartado se trata es de la autoficción en grado cero, o de cuando el autor adopta el disfraz del crítico, lo cierto es que Robbe-Grillet no tuvo nunca acceso al texto del autor de La Recherche disfrazado del Otro, travestido en crítico, que, de forma no declarada, había hecho la crítica de la propia obra. La explicación es simple, de pura cronología: en el 2014, año de publicación por vez primera de L'esthétique de Marcel Proust, hacía seis años que Robbe-Grillet había fallecido. Así que sucede aquí, como en tantas otras ocasiones, que el intertexto se debería más al azar objetivo que a la relación causal de la influencia...

\section{4.- 3.- ALAIN ROBBE-GRILLET}

Robbe-Grillet, igual que Proust, pero sin haber tenido noticia de aquella actividad como crítico de sí mismo, escribe el texto que lleva por título Un écrivain non réconcilié, texto 
de crítica - de teoría- sobre su propia obra, ocultando la identidad de la autoría del discurso crítico y refiriéndose a Robbe-Grillet en tercera persona, con el desparpajo del crítico que designa al escritor objeto del estudio, en modo de fingimiento o, si se quiere, de lo que aquí se ha dado en nombrar autoficción en grado cero.

Si en el caso de L'esthétique de Marcel Proust se consideró conveniente recordar los avatares de su gestación, parece ahora oportuno ocuparse también de la contextualización de Un écrivain non réconcilié y evocar brevemente su particular historia. Este texto se publica por vez primera en 1972, como prefacio a la edición de bolsillo de la colección 10/18 de la novela de Robbe-Grillet La maison de rendez-vous (novela que ya había sido publicada originalmente en la edición blanca de Minuit en 1965). El texto está firmado por un tal Franklin J. Matthews, cuyo nombre aparece en la misma portada de esa edición de la novela como autor de un prefacio, que dos páginas más adelante será calificado de inédito. La tradición oral de la crítica especializada había tenido por establecido que Franklin J. Matthews era un crítico californiano, profesor de una universidad australiana. Una segunda publicación del texto, en 1978, dentro del número monográfico de la revista Obliques ( $n^{\circ}$ 16-17) dedicado a RobbeGrillet, continúa dentro del mismo sigilo y atribuye el texto al mismo Franklin J. Matthews. Habrá que esperar al primer año del siglo siguiente, al año 2001, para que el secreto sea revelado en la nota que acompaña a la tercera publicación del texto Un écrivain non réconcilié en la página 95 de Le Voyageur. En su correspondencia con Catherine, en octubre de 1971, Alain Robbe-Grillet había escrito el fragmento que se reproduce a continuación, no exento de una buena carga de ironía, y hasta con alguna que otra deformación humorística de la grafía: «Ce jour d' hui, le x octobre 71 au Mesnil, je, Alain, certifie sur l' honneur que mon immarcescible et cristalline épouse a lu, d'un bout à l'autre et avec soin -et avec des commentaires intelligents et zélogieux- l'article du professeur Matthews arrivé le matin même des Nouvelles Galles du Sud », firmado con una simple A, sería publicado cuatro años después de su fallecimiento, en el 2012 (Alain et Catherine Robbe-Grillet: 350).

Desde el primer párrafo el texto Un écrivain non réconcilié insiste en presentarse como un mensaje dirigido a una crítica que suele reprochar al autor la contradicción con las obras anteriormente publicadas y con las teorías sobre la novela que él mismo había expuesto:

Lors des nombreuses interviews qu'il accorde à la presse pour la sortie de chaque nouveau livre, Alain Robbe-Grillet insiste volontiers sur la continuité des recherches romanesques qu'il poursuit depuis bientôt vingt ans. Pourtant la critique littéraire, unanime sur ce point, semble avoir une opinion tout opposée : c'est, au contraire, des ruptures incessantes qu'elle se plait à signaler, les ennemis 
en faisant à l'auteur un de leurs griefs (lui reprochant à chaque fois de renier ses œuvres passées comme ses propres théories) (Robbe-Grillet, 2001 : 95).

El camino que emprende el pretendido crítico californiano, profesor de una universidad australiana, es el de poner de manifiesto la depravación de los temas que aparecen en las últimas ficciones de Robbe-Grillet, tanto en la literatura como en el cine, para insistir en que se trata de la expresión de una mitología característica del mundo contemporáneo y de la fantasía del autor (y de sus fantasmas), que han comenzado a manifestarse de modo más evidente, sin que ello constituya una auténtica ruptura con la obra precedente; más bien se diría que la continúan. Sus dos últimas novelas, La maison de rendez-vous y Projet pour une révolution à New-York (1970), trabajarían a partir de lo que se denomina «un material sin alma» y se ocuparían de objetos de pacotilla alejados de los grandes temas de la tradición humanista, en la línea, en cierto modo de lo que el propio autor había calificado de «mis temas

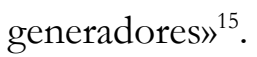

El estudio de la significación del erotismo y de la inmovilidad dentro de las ficciones de Robbe-Grillet ocupan un lugar destacado en el trabajo del «crítico australiano». Las jóvenes dentro de un escenario íntimo, como forma de representación inmóvil del objeto de deseo, contempladas por el ojo del narrador de modo preciso y minucioso, constituyen la mejor muestra de las virtudes paralizantes de una escritura que se compara con la flecha de Zénón de Elea. El movimiento necesario para la producción del conflicto requerido por la actividad narrativa viene dado por las figuraciones varias de la violencia: «Écriture mouvante autour d'un sujet immobile [...] entre l'écriture et le thème qu' elle développe, entre le thème développé et celui [...] dont on pressent la déflagration toute proche (brisure, cri strident, déchirure, écroulement, explosion, assassinat). Et en même temps le caractère inaltérable des choses continue à s'imposer: la flèche de Zénon poursuit imperturbablement son vol immobile ». Escritura, pues, esencialmente de naturaleza paradójica, condenada a vagar entre la inmovilidad y la violencia transformadora, a través de la representación del fantasma al objeto de crear la fantasía ficcional..., como el movimiento continuado de las olas que

\footnotetext{
15 «Couvertures illustrées des romans qu'on vend dans les gares, affiches géantes, revues pornographiques des sex-shops, publicités vernies des magazines de mode, figures peintes à plat des bandes dessinées, autrement dit tout ce qui a remplacé ma prétendue profondeur, ce moi qui a été chassé de mon for intérieur et se trouve aujourd'hui placardé en pleine lumière dans les vitrines ou dans les murs mêmes de la cité». Intervención de Robbe-Grillet «Sur le choix des générateurs», en el coloquio de Cerisy sobre el Nouveau Roman en julio de 1971, publicada en Nouveau Roman: hier, aujourd'bui, UGE, 10718, 1972, tome 2, pp. 157-162.
} 
rompen en la playa, sin que la subida o la bajada de la marea sea del todo perceptible a los ojos del espectador apostado en la orilla.

Un écrivain non réconcilié está dividido en cuatro apartados, al ya mencionado « $\mathrm{La}$ dépravation des thèmes » siguen «Surface et profondeur», «L'immobile et l'inaltérable » $\mathrm{y}$ 《 Le conflit dans l'écriture comme générateur du roman». Franklin J. Matthews-Robbe-Grillet lo que ha escrito no es solamente un prefacio a la edición de una de sus novelas, sino una teoría bien construida que permitiría encuadrar no sólo a esa novela sino al conjunto de novelas de la denominada segunda época o del nouveau nouveau roman, que incluye desde La maison de rendez-vous y Projet pour une révolution à New-York hasta La reprise (2001) y Un roman sentimental (2007), pasando por Topologie d'une cité fantôme (1976), Souvenirs du triangle d'or (1978) y Djinn (1981). A diferencia de Marcel Proust, que adopta el disfraz del crítico al final de la escritura de su obra, Robbe-Grillet lo hace in medias res, con las consecuencias determinantes que ello haya podido suponer para las lecturas de otros críticos y la recepción de la obra a partir del inicio de la década de los años setenta.

El nombre de Franklin. J. Matthews bien podría designar a un crítico australiano, pero cuando se mira de cerca, resulta que sus componentes grafemáticos tienen relación directa con la onomástica específica del autor en Le voyeur y La jalousie, donde los personajes se llamaban respectivamente Mathias y Frank.

\section{5.- LA ESCRITURA DE LA TEORÍA EN LA NUEVA AUTOBIOGRAFÍA: LES} ROMANESQUES, LA AUTOFICCIÓN EN PRIMER GRADO.

La trilogía de Alain Robbe-Grillet Les romanesques se publica entre 1984 y 1994. Escritura autobiográfica, autoficción con distancias respecto a los géneros establecidos, al amparo de lo que el autor ha denominado nouvelle autobiographie, del mismo modo que la categoría del nowveau roman trataba de evocar la distancia con relación a los parámetros genéricos establecidos por la tradición de la novela... El binomio proximidad-distancia en estos textos de Robbe-Grillet se revela especialmente rentable a efectos de lectura, con relación al género y con relación a la persona del autor. El lector de esta trilogía quedaría absolutamente decepcionado si lo que busca en ella es el relato de una vida, la biografía, por fin, de un autor tildado de objetivista y hasta del crimen de lesa humanidad, y sin embargo hay pasajes ciertamente cercanos... El término de autoficción sería más adecuado, al sugerir 
la representación del autor a través de un personaje, de novela o novelado, pero no del todo, porque la palabra del narrador va y viene como sujeto y objeto de la narración, unas veces como palabra del autor que es Robbe-Grillet y otras con focalización directa sobre el personaje Henri de Corinthe. Es bien conocido que Corinthe es un lugar por excelencia de pérdida de la identidad en la tradición literaria, es la ciudad de Edipo, que no era de allí, y Edipo, o al menos el texto de Sófocles, desempeñaba una función de primer orden desde Les Gommes (1953). Roger-Michel Allemand (1991) ha estudiado de forma detallada las duplicaciones y la duplicidad en Les romanesques, dedicando atención particular a los dobles del personaje narrador y narrado: ambigüedad histórica del personaje de Corinthe, sus relaciones enigmáticas con el padre del narrador, su estatuto como doble del padre y del propio narrador, en la medida en que no sea esa figura paterna la representación del padre de Chateaubriand, como Robbe-Grillet ha pretendido en más de una ocasión.

Uno de los objetivos -ni único, ni probablemente prioritario- de Le miroir qui revient (1984), la primera de Les romanesques, es una toma de posición de carácter teórico, desde la propia praxis de la escritura, contra las aserciones contenidas en Le pacte autobiographique de Philippe Lejeune (1975), en el que se daba por establecida la identidad de autor, narrador y personaje dentro del género autobiográfico. En alguna medida la relación identitaria de estas tres instancias salta por los aires dentro de un texto construido de manera conflictiva, articulado, como se dijo arriba por el binomio proximidad-distancia ${ }^{16}$. El término «autobiographie», con varias ocurrencias dentro del relato, es utilizado por el narrador en contextos o comentarios de orientación metatextual, restrictivos y, en todos los casos, distantes:

J'éprouve aujourd'hui un certain plaisir à utiliser la forme traditionnelle de l'autobiographie (Robbe-Grillet, 1984 : 16).

Quant au moi, de tout temps haïssable, il prépare sans aucun doute une rentrée en scène encore plus frivole : celle du biographisme (Robbe-Grillet, 1984 : 10). Je tâtonne encore ici, par perversité, dans l'entreprise réaliste, biographique et représentative (Robbe-Grillet, 1984 : 30).

À qui veut l'entendre, j'affirme récuser l'entreprise autobiographique (RobbeGrillet,1984 : 58).

La forma fragmentaria en la que se presenta Le miroir qui revient colabora a la producción de un efecto de distancia. Se alterna el discurso metatextual de orientación 
interna, referido al propio relato, y el intertextual de orientación restringida (las otras obras del mismo autor) y general (los otros autores presentes en el texto, y entre ellos al que se refiere la exclamación casi final «Flaubert c'est moi!»), con los discursos de la teoría, la ficción y la narración de aventuras personales y familiares, teniendo, en ocasiones, como fondo los marcos históricos del nazismo, la Ocupación o la Segunda Guerra Mundial.

El vaivén del espejo en medio de las olas del mar - alternativamente se acercan y se alejan de la playa-, en el episodio del espejo que vuelve y que sirve para titular el texto entero, bien puede tomarse por movimiento modélico a efectos de la estructuración del propio relato (especie de mise en abyme temático-estructural) mediante el cual la paradoja de la autobiografía exhibiría la contradicción de su funcionamiento: la ficción es responsable de la estructura de una narración que no tiene más aval de autenticidad que el imaginario, fantasmagórico y legendario. El narrador del texto, por su parte, hace los mismos esfuerzos que su doble de ficción: «Corinthe s'y cramponne de toutes ses forces [...] Au prix d'efforts démesurés, il finit pourtant par venir à bout de ce labeur absurde auquel il s'est senti contraint » (RobbeGrillet, 1984: 93). Las características del relato del personaje Henri de Corinthe, en el delirio sufrido tras el episodio del espejo que vuelve, pueden ser compartidas en la misma medida por el narrador de Le miroir qui revient:

Une des particularités de son récit, qui en rendait le déroulement quasiment impossible à suivre, outre sa fragmentation excessive, ses contradictions, ses manques, ses redites, le fait qu'il y mélangeait constamment les temps du passé avec des brusques passages au présent qui paraissait pourtant concerner la même période de sa vie, et les mêmes événements (Robbe-Grillet, 1984 : 98).

La tentación de aplicar a este libro de Robbe-Grillet lo que el ya varias veces citado en este trabajo Roland Barthes escribiera en su Roland Barthes par Roland Barthes es difícil de superar, porque en uno y en otro caso el imaginario es obra de varias máscaras (personae). Uno y otro libro progresan a base de soplos de la imaginación y de punzadas críticas: «Pas de plus pur imaginaire que la critique (de soi). La substance de ce livre, finalement, est donc totalement romanesque » (Roland Barthes, 1975 : 123-124).

El lector de Les romanesques se topará con dos tipos de discursos críticos, uno referido a la teoría de la literatura (de la escritura) en general y otro a la historia de la literatura francesa, y en particular a la del nouveau roman. No conviene perder de vista que en esos tres libros un objetivo esencial es el de trazar un retrato de la persona del escritor, definida en 
estos términos por el narrador: «Une personne [...] est à la fois un corps, une projection intentionnelle et un inconscient» (Robbe-Grillet, 1984: 12).

Los discursos sobre el inconsciente, la perspectiva freudiana del psicoanálisis, no son ajenos a las inquietudes en materia de teoría sobre la escritura de Robbe-Grillet. Sin ánimo de extendernos aquí sobre un aspecto que merecería y ha merecido desarrollos particulares, me conformaré, dentro del corpus de Les romanesques, con evocar con la brevedad requerida dentro del espacio de un artículo como éste, lo que se cuenta en las páginas de clausura de la segunda de ellas, Angélique on l'enchantement. El relato de las diez últimas páginas tiene de qué sorprender para quienes se dedican al rastreo de los mitos personales y de las metáforas obsesivas ${ }^{17}$, que bien pudieran tomar por relato de una escena primigenia y expresión pura del origen del fantasma profundo. En ellas se cuenta una vivencia del autor adolescente de carga potencialmente traumática, o, en todo caso, con la capacidad suficiente para impresionar a un muchacho de corta edad (trece años). El narrador trae a colación el regreso en coche a Paris, después de una pequeña excursión, en compañía de Catherine, su mujer, y de Jérôme Lindon, su editor, mencionado en las páginas anteriores de este trabajo. El relato tiene como destinario principal a Jérôme Lindon, y a él le dice que la joven-personaje de Le voyeur ha existido, «comme d'ailleurs tout ce qui se trouve dans mes livres, qu' elle ne s'appelait ni Violette ni Jacqueline, mais Angélique, et que je dirais peut-être un jour sa vraie histoire. Le ferai-je ? » (Robbe-Grillet, 1987). La «verdadera historia» pudiera ser, según se lee en el texto, la de una niña de doce años que un día aparece muerta en el mar, junto al acantilado de la costa bretona. Compañera de juegos prohibidos del narrador, adolescente como ella, ejerce sobre él una particular forma de poder y le inicia de forma perversa en el sexo, induciéndole a confundir la sangre de las primeras reglas y la de la desfloración... El crítico avezado en la lectura de la obra de Robbe-Grillet, literaria y fílmica, tiene en ese relato que ocupa las últimas páginas de Angélique ou l'enchantement materia de la que ocuparse, pero bien haría en preguntarse si no será esa la trampa mejor urdida por un escritor hacia el discurso crítico que es capaz de generar, teniéndolo previsto, adelantándose a él, para negarlo, o simplemente ponerlo en tela de juicio; al fin y a la postre Angélique es un personaje de ficción y el libro una especie de novela, porque desde la escritura « la chair des phrases est comme celle des femmes ».

${ }^{17}$ Fernández Cardo: 1987. 
El primer párrafo de la tercera de Les romanesques, Les derniers jours de Corinthe (1994), es particularmente significativo al objeto de la aproximación de escritura y erotismo, que acaba de ser sugerida. El lector juzgará por si mismo -me he permitido traducir el texto, porque esta tercera parte de la trilogía no ha sido publicada en español- sobre la confluencia de algunos de los temas tratados, redundantes, a lo largo de este trabajo:

La carne de las frases ha ocupado siempre, sin duda, un lugar importante en mi trabajo. Incluso cuando no estoy en mi mesa sus figuras movedizas no dejan de obsesionarme. Repito palabras, ritmos, pruebo sonoridades, organizo ecos y rupturas. Está en mí como los movimientos repetidos, previsibles, sin cesar imprevistos, del agua profunda que abraza, abofetea, sumerge, desnuda de un solo golpe hasta los pies, luego acaricia dulcemente las rocas de granito rosa, brillantes, pulidas por los remolinos con franjas de espuma (Robbe-Grillet, 1994).

Y lo que sigue, el párrafo segundo, continúa insistiendo en el mismo registro al evocar las manos que abrazan la materia misma del lenguaje, su prosodia, su textura, que «presenta con claridad ante todo un carácter sensual». El lenguaje produce una música como la del mar cuando golpea el antiguo suelo duro de Bretaña y trae a la memoria a Breton, Tristan Corbière, Valéry y Lautréamont (y la exclamación de éste, iyo te saludo, viejo Océano!)... Una vez más asistimos a la reunión del movimiento de la escritura y el de las olas del mar, al que la reescritura de la primera frase de La maison de rendez-vous «La chair des femmes a toujours occupé, sans doute, une grande place dans mes rêves» (Robbe-Grillet, 1965) no dejará de alimentar con su propia carga de significación erótica desde el lado de la ensoñación ficcional.

\section{6.- ROBBE-GRILLET ESCRITOR OBJETO DE LA NARRACIÓN: MARIO BELLATIN,} LA AUTOFICCIÓN EN SEGUDO GRADO.

Si se consideran ecuaciones de segundo grado aquellas en la que la incógnita aparece al menos una vez elevada al cuadrado, no habría gran dificultad en servirse de la expresión matemática «en segundo grado» para nombrar el tipo de autoficción desarrollado en el relato de Mario Bellatin En el ropero del señor Bernard falta el traje que más detesta, que ocupa la mitad de un libro cuyo título general es Gallinas de madera (2013). La incógnita del narrador de la autoficción se eleva al cuadrado en la medida en que se produce la identificación de éste con otro personaje que toma la palabra por él, le dicta el pensamiento, le escribe ficciones 
imaginarias, hasta que lisa y llanamente no hay separación de uno y otro y el texto progresa en primera persona del plural a través del pronombre «nosotros».

Si el yo inicial del relato debe tener como referente, puesto que de autoficción se trata, el nombre del autor que figura en la portada, o sea Mario Bellatin, el personaje que, en principio, se presenta como objeto para el sujeto narrador es el señor Bernard, que de ese modo se le designa a lo largo de todo el texto. Desde la contraportada, el lector que tiene el libro en sus manos recibe la información de que en realidad el señor Bernard no es otro que el escritor Alain Robbe-Grillet: «Bellatin narra sus paseos con el señor Bernard -trasunto de Robbe-Grillet-, con quien Mario Bellatin sostuvo uno de sus últimos diálogos públicos antes de su muerte».

No entraremos en el detalle de si esa información histórica suministrada desde la contraportada es rigurosamente cierta; no parece que ese fuera el último diálogo público mantenido por Robbe-Grillet antes de su muerte, acaecida en 2008. Sí es cierto, que Mario Bellatin mantuvo en México, con toda probabilidad en la última década del pasado siglo ${ }^{18}$, un encuentro con el novelista francés, y que el propio Bellatin refiere en una entrevista publicada en la revista Replicante, periodismo digital, cultura crítica en octubre de 2013, cuando realizaba la promoción mediática de su nuevo libro:

Ciertamente tuve un encuentro fortuito con Robbe-Grillet. Un día me invitaron a conocerlo justo cuando estaba en México. jImaginate!, yo pensaba que ya estaba muerto... Al parecer los franceses viven mucho. Para mí fue una gran sorpresa. Él había sido importante porque cuando yo escribía en los años ochenta (años oscuros para la narrativa latinoamericana) muchas instancias, entre ellas la crítica, estaban basadas en verdades preconcebidas, cánones, caminos y formas que se tenían que seguir de manera obligatoria. Yo me decía: «iNo es posible!, si esto es un arte hay que considerar la libertad como primer requisito». Y una de las historias de Gallinas de madera, la que tiene que ver con él, es precisamente la conversación que sostuvimos. Bernard es Robbe-Grillet y mucho de las ideas que planteo en el libro son las que él me dijo, y los autores que señalo son autores reales, sólo que están sin nombre.

Ciertamente el único nombre propio de escritor que aparece en el relato es el fingido de Bernard (además del nombre de Mario Bellatin). Ni siquiera se nombra el movimiento del nouveau roman, siempre reemplazado en todas las ocurrencias esperables por la denominación de «Movimiento Literario Sumamente Innovadon». Corresponde al lector el papel de identificar a través de los elementos que el texto va proporcionando el nombre de los autores

${ }^{18}$ En Alain Robbe-Grillet. Le voyageur du nouveau roman, chronologie illustrée 1922-2002, Éditions de l'IMEC, Paris, 2002, se señala como fecha de su viaje a México la de noviembre de 1991. 
aludidos: Nathalie Sarraute, Claude Simon, Marguerite Duras, Jean Paul Sartre, Albert Camus e incluso Chateaubriand. Bien podría ser utilizado el texto de Bellatin como propuesta de examen para los alumnos de una asignatura como Literatura Francesa Contemporánea: la calificación final en directa dependencia del número de los aciertos.

Las teorías expuestas por el señor Bernard-Robbe-Grillet a propósito de la literatura y de la autobiografía han sido determinantes para el interlocutor sujeto enunciador de la autoficción: «las palabras del señor Bernard me hicieron encontrar otra forma de escritura» (Bellatin, 2013: 112). El señor Bernard reprocha a los críticos literarios su confianza en algunas tonterías: «Se sabe que los científicos no creen en la misma ciencia» (Bellatin, 2013: 124). El narrador, imbuido de las ideas de su interlocutor, se muestra convencido de que la autobiografía tiene todo el derecho de inventar: «La regla era que la autobiografía debía ser sincera y verídica, y yo no creía que eso fuera posible [...]Creo que la autobiografía tiene todo el derecho de ser solamente ella misma. De crear cosas que le atañen solo a ella. No únicamente cuenta con el derecho, sino también tiene el deber de que así sea» (Bellatin, 2013: 129).

En el relato de Bellatin hay sólo un nombre propio de personaje que se cita, precisamente el de Henri de Corinthe, que es el personaje de Les romanesques, y que fue aludido antes, en este trabajo, como pieza fundamental de las duplicidades en el texto de los tres volúmenes de la nouvelle autobiographie de Robbe-Grillet. Mientras algunos críticos han utilizado el término de esquizofrenia para explicar su proceso de elaboración, en el texto de Bellatin todo se resuelve de manera mucho más sencilla, lo reduce al nivel de la escritura de un fantasma personal de la infancia:

El conde Henri de Corinthe existió, pero yo no sé absolutamente nada de él, no tengo más que escasos testimonios huellas de su existencia que me quedaron en la historia de Francia porque él fue un personaje sobre el que se ha fantaseado mucho $[. .$.$] me encerraban en mi cuarto del primer piso cuando mi padre y el$ conde Henri de Corinthe hablaban. Una noche bajé las escaleras y me puse a escuchar detrás de la puerta [...] Reconocí la voz de mi padre y otra voz que era también la de mi padre. En ese momento, como era un niño inclinado a lo fantástico, me dije, ah sí, es por eso que no me dejan bajar, porque no quieren que presencie este acontecimiento perturbador. Algunas tardes mi padre se desdoblaba y mantenía grandes conversaciones consigo mismo [...] Se puede encontrar un aspecto de esto en algunas partes de mi escritura. Cualquiera sabe, es una tesis generalmente aceptada, que toda la obra de un escritor se forma en la infancia (Bellatin, 2013: 130-131). 
Bien pudiera ocurrir que el relato En el ropero del señor Bernard falta el traje que más detesta estuviera dotado de la capacidad de proporcionar a los interesados una excelente guía de introducción a la lectura de la obra de Robbe-Grillet en su conjunto ... El momento ha llegado de preguntarse si en la reunión mantenida con el escritor mexicano Robbe-Grillet le hizo confidencias extraordinarias e inusuales sobre la literatura, la novela y la autobiografía. No es propósito de estas líneas decepcionar al lector, sino incrementar su interés, porque si es cierto que la mayor parte de las ideas expuestas en el texto de Bellatin constituyen algunos de los lugares comunes diseminados en las entrevistas y declaraciones del novelista francés, no lo es menos que sobre todo proceden de la escritura del propio Robbe-Grillet en los tres volúmenes de Les romanesques. ¿Ante qué tipo de texto estamos entonces, cuando la autoficción de Bellatin reescribe la autoficción de Robbe-Grillet? ¿Qué tipo de literatura resulta de la escritura conjunta de la ficción y de la teoría cuando además la incógnita identitaria está elevada al cuadrado? Sin duda «hay una gran resistencia a aceptar una escritura semejante» (Bellatin, 2013: 136), no en menor grado que la que en su momento despertara la escritura proustiana.

\section{7.- A MODO DE CODA: DOBLE O NADA, UNA APORÍA DE ZENÓN DE ELEA.}

Cécile Voisset-Veysseyre plantea una lectura global de la obra de Robbe-Grillet que se sitúa a partir de la ruina de los procesos de representación y de identidad: «cela reviendrait à savoir si le sujet, défini par son image unifiée dans le miroir, n’est pas en voie de disparition selon le texte robbe-grilletien qui s'essaie à le gommer» (2011: 152). La imagen del doble, tal como el propio Robbe-Grillet se ha encargado de repetir en ocasiones varias, es uno de sus fantasmas recurrentes ${ }^{19}$, que va a contribuir a la disolución de la identidad. De este modo la lectura va a verse obligada a transitar por espacios recurrentes, repetitivos en apariencia, pero sin volver exactamente al lugar de partida, dentro de un juego de combinaciones que permiten avanzar al relato. En un tipo de texto en que el discurso del autor está roto, el asunto del doble $e^{20}$ aparece frecuentemente tematizado mediante el personaje del espía y su contrario (contraespionaje), el agente doble. Estas y otras consideraciones de parecido calado

\footnotetext{
${ }^{19}$ Robbe:Grillet, 2001: 271-273, « Mon double coréen » [1997].

${ }^{20}$ El trabajo de Lourdes Carriedo «Aspectos del doble en Djinn de Alain Robbe-Grillet: el juego de la duplicidad y ambigüedad narrativas», Cédille, Revista de Estudios Franceses, Monografías 2, 2011, aborda en detalle (estudio del texto de la novela Djinn) algunas de las cuestiones que aquí sólo se suscitan de manera breve.
} 
hacen que el texto de Robbe-Grillet sea calificado como doblemente lúdico: «il est un jeu qui ne se prête à une lecture référenciante, il est un je qui ne se prête à une lecture unifiante. Je n'est pas donc un autre, car il est tout autre; son pluriel est celui du double » (VoissetVeysseyre, 2011: 162).

Planteada la cuestión de la escritura robbe-grilletiana en términos tales, salta a la vista la imposibilidad de separar los discursos de la teoría y de la ficción (autoficción incluida, naturalmente). La noción de aporía, entendida como dificultad sin salida, se instala, pues, en el centro de una lectura que buscaría de nuevo el modelo de funcionamiento en una de las de Zenón de Elea, en esta ocasión la conocida con el nombre de «Aquiles y la tortuga». Basta con identificar a Aquiles con el discurso de la teoría y a la tortuga con el de la ficción, o viceversa -ya que de juego de dobles se trata-, a sabiendas de que el desplazamiento casi imperceptible de ésta no podrá ser superado -ad infinitum- por la carrera brillante del gran corredor del Ática, dentro de una práctica de escritura abocada a la inmovilidad repetitiva. Franklin J. Matthews, el escritor no reconciliado, y Zenón confluyen así en una misma actitud, «eminentemente polémica y crítica» ${ }^{21}$. 


\section{BIBLIOGRAFÍA}

Allemand, Roger-Michel (1991): Duplications et duplicité dans les «Romanesques» d'Alain RobbeGrillet, Paris, Lettres Modernes.

Barthes, Roland (1963): «Préface» en Bruce Morrissette: Les romans de Robbe-Grillet, Paris, Les Éditions de Minuit: 7-16.

Barthes, Roland (1966): Critique et verité, Paris, Éditions du Seuil.

Barthes, Roland (1975): Roland Barthes par Roland Barthes, Paris, Éditions du Seuil.

Bellatin, Mario (2013): Gallinas de madera, Madrid-México, Editorial Sexto Piso.

Bueno, Gustavo (1974): La metafísica presocrática, Oviedo, Pentalfa Ediciones.

Carriedo López, Lourdes (2011): «Aspectos del doble en Djinn de Alain Robbe-Grillet: el juego de la duplicidad y ambigüedad narrativas», Cédille, Revista de Estudios Franceses, Monografías 2: 149-174.

Corpet, Olivier, et Lambert, Emmanuelle (2002): Alain Robbe-Grillet le voyageur du nouveau roman. Chronologie illustrée 1922-2002, Paris, Éditions de L'IMEC.

Coste, Didier (1988): «Pour une histoire littéraire négative», Actes Noésis II. L’internationalité littéraire, París-Barcelona, Noesis.

Deleuze, Gilles (1969): Logique du sens, Paris, Les Éditions de Minuit.

Fernández Cardo, Jose María (1985): «Autobiografía y distancia», Noesis 3: 81-90.

Fernández Cardo, Jose María (1994): «La autobiografía que vuelve: Robbe-Grillet», Barcarola, 44-45: 257-269.

Lanfried, Carrie; Olivier Wagner (eds.) (2021): Nouveau roman. Correspondance.1946-1999, Gallimard.

Lejeune, Philippe (1975): Le pacte autobiographique, Paris, Seuil.

Matthews, J. Franklin (1972): «Préface» en La maison de rendę-vous, Paris, Union Générale d' Éditions, Coll.10/18.

Meschonnic, Henri (1970): Pour la poétique I, Paris, Gallimard, 1970.

Pereira Romero, Teresa (1993): Silencio y poesía. La obra de Yves Bonnefoy, Oviedo, Servicio de Publicaciones de la Universidad

Robbe-Grillet, Alain (1953): Les gommes, Paris, Les Éditions de Minuit.

Robbe-Grillet, Alain (1955): Le voyeur, Paris, Les Éditions de Minuit.

Robbe-Grillet, Alain (1957): La jalousie, Paris, Les Éditions de Minuit. 
Robbe-Grillet, Alain (1963): Pour un nouveau roman, Paris, Les Éditions de Minuit.

Robbe-Grillet, Alain (1965): La maison de rendez-vous, Paris, Les Éditions de Minuit.

Robbe-Grillet, Alain (1970): Projetpour une révolution à New-York, Paris, Les Éditions de Minuit.

Robbe-Grillet, Alain (1981): Djinn, Paris, Les Éditions de Minuit.

Robbe-Grillet, Alain (1984): Le miroir qui revient, Paris, Les Éditions de Minuit.

Robbe-Grillet, Alain (1988): Angélique ou l'enchantement, Paris, Les Éditions de Minuit.

Robbe-Grillet, Alain (1994): Les derniers jours de Corinthe, Paris, Les Éditions de Minuit.

Robbe-Grillet, Alain (2001): La reprise, Paris, Les Éditions de Minuit.

Robbe-Grillet, Alain (2001): Le voyageur. Textes, causeries et entretiens (1947-2001), Christian Bourgeois Éditeur.

Robbe-Grillet, Alain (2005): Préface à une vie d'écrivain, Seuil, France Culture.

Robbe-Grillet, Alain (2007): Un roman sentimental, Fayard.

Robbe-Grillet, Alain et Catherine (2012): Correspondance, 1951-1990, Fayard.

Robbe-Grillet, Catherine (2012): Alain, Fayard.

Schneider, Michel (2014): L'auteur, l'autre. Proust et son double, Gallimard.

Simonin, Anne (2000): «La mise à l'épreuve du nouveau roman. Six cent cinquante fiches de lecture d'Alain Robbe-Grillet (1955-1959)», Annales. Histoire, Sciences Sociales, 55 année, 2: 415-437.

Voisset-Veysseyre, Cécile (2011): «Quitte ou double? Robbe-Grillet et le mythe de l'identité», Amaltea, Revista de Mitocrítica, 3 : 151-165. 
SOBRE EL AUTOR

\section{José María Fernández Cardo}

Profesor de Literatura Francesa de la Universidad de Oviedo.

Investigación: Literatura Francesa de los siglos XIX y XX. Literatura comparada e intertextualidad.

ORCID: 0000-0003-2566-0040

Contact information: correo electrónico: cardo@uniovi.es 